Meta

Journal des traducteurs

Translators' Journal

\title{
Comment peut-on être sourcier ? Critique du littéralisme en traduction
}

\section{Jean-René Ladmiral}

Volume 62, numéro 3, décembre 2017

La traduction littéraire comme création

URI : https://id.erudit.org/iderudit/1043947ar

DOI : https://doi.org/10.7202/1043947ar

Aller au sommaire du numéro

\section{Éditeur(s)}

Les Presses de l’Université de Montréal

ISSN

0026-0452 (imprimé)

1492-1421 (numérique)

Découvrir la revue

Citer cet article

Ladmiral, J.-R. (2017). Comment peut-on être sourcier ? Critique du littéralisme en traduction. Meta, 62(3), 538-551. https://doi.org/10.7202/1043947ar

\section{Résumé de l'article}

La prise en compte de la traduction littéraire comme création pose d'emblée l'immémoriale question du littéralisme, qui oppose sourciers et ciblistes. Quand on traduit une oeuvre littéraire, le théorème de dichotomie met en évidence le choix à opérer entre la spécificité ethnoculturelle (et linguistique) du texte original et l'esthétique littéraire qui est la sienne, et au sein de laquelle le rythme est un aspect parmi d'autres. Or il apparaît que l'accent mis trop souvent sur le décalage interculturel est plus ou moins surdéterminé par des impensés idéologiques. Mais la créativité littéraire de la traduction est un enjeu esthétique, et non pas idéologique. Cela dit, l'idée même de créativité est tendanciellement aporétique - et ce, alors qu'elle est concrètement à l'oeuvre dans les traductions de haut niveau. 


\title{
Comment peut-on être sourcier? Critique du littéralisme en traduction
}

\author{
JEAN-RENÉ LADMIRAL \\ Université de Paris Ouest-Nanterre et ISIT, France
}

\section{RÉSUMÉ}

La prise en compte de la traduction littéraire comme création pose d'emblée l'immémoriale question du littéralisme, qui oppose sourciers et ciblistes. Quand on traduit une œuvre littéraire, le théorème de dichotomie met en évidence le choix à opérer entre la spécificité ethnoculturelle (et linguistique) du texte original et l'esthétique littéraire qui est la sienne, et au sein de laquelle le rythme est un aspect parmi d'autres. Or il apparaît que l'accent mis trop souvent sur le décalage interculturel est plus ou moins surdéterminé par des impensés idéologiques. Mais la créativité littéraire de la traduction est un enjeu esthétique, et non pas idéologique. Cela dit, l'idée même de créativité est tendanciellement aporétique - et ce, alors qu'elle est concrètement à l'œuvre dans les traductions de haut niveau.

\section{ABSTRACT}

Any consideration of literary translation as a creation begs the age old question of literalism that opposes source-oriented and target-oriented translators. In translating a literary work, the postulate of dichotomy indicates the operative choice between the ethnocultural (and linguistic) specificity of the original text and its inherent literary esthetic, within which textual rhythm is a central aspect among others. It seems that the insistance that is too often put on the intercultural gap is more or less overly determined by ideological unthoughts. But the literary creativity of a translation is an esthetic and not an ideological strategy. Having said this, the very idea of creativity in this context is an aporetic tendency, although it is definitely implemented in superior translations.

\section{RESUMEN}

Considerar la traducción literaria como una creación plantea desde el principio la cuestión inmemorial de la literalidad o la oposición entre sourciers y ciblistes. Al traducir una obra literaria, el teorema de dicotomía pone de manifiesto la decisión que se debe tomar entre la especificidad etnocultural (y lingüística) del texto original y su estética literaria propia, dentro de la cual el ritmo es tan solo un aspecto. Ahora bien, se observa que la insistencia, a menudo excesiva, en el desfase intercultural está más o menos sobredeterminada por impensados ideológicos. Sin embargo, la creatividad literaria de la traducción es un desafío estético y no ideológico. Dicho esto, el propio concepto de creatividad tiende a ser aporético, pese a estar presente de forma concreta en las traducciones de alta calidad.

\section{MOTS CLÉS/KEYWORDS/PALABRAS CLAVE}

littéralisme, esthétique, idéologie, créativité littéraire

literalism, esthetics, ideology, literary creativity

literalidad, estética, ideología, creatividad literaria 
à la regrettée Claudette Oriol-Boyer

I.

Je commencerai en modulant mon propre titre. Le premier membre y est une question rhétorique, où l'interrogation rejoint l'exclamation: "Mais comment donc peut-on être sourcier?!» La question, à dire sur un ton quasiment scandalisé, comme la réaction à une absurdité qui saute aux yeux, appelle une réponse négative qu'énonce le deuxième membre du même intitulé: la critique du littéralisme, c'est-à-dire la critique des «sourciers» que j'oppose aux ciblistes - sur quoi je vais revenir.

En attendant, l'intitulé général du colloque ${ }^{1}$ nous appelle à poser le problème de la créativité en traduction. J'y distinguerai trois facettes: a) la créativité a contrario ou le déni apparent de la créativité; b) les dessous de la créativité, qui cachent d'abord ce qu'elle finit par laisser voir...; c) enfin, l’horizon aporétique de la créativité.

Quant aux deux concepts de sourcier et cibliste, j'en avais campé l'opposition lors d'un colloque à Londres le 18 juin 1983 ; puis, ils m’ont échappé et se sont répandus comme une traînée de poudre, avant de déclencher des polémiques qui m’ont surpris et m'ont amené à m'engager dans de nombreux débats. Au bout du compte, tout cela a fini par fournir la matière d'un livre, tant il est apparu combien cette problématique a été toujours présente en traduction (et, peut-être plus encore, en théorie de la traduction) et combien elle recèle des enjeux qui vont bien au-delà de la traduction elle-même (Ladmiral 2015). Cela concerne bien sûr la traduction littéraire, qui reste le cœur du projet traductif, avec la part de création qui lui est inhérente; mais il m'est apparu aussi que cette question immémoriale du littéralisme débouche sur plus d'une douzaine de problématiques tout à fait actuelles, dont certaines seront évoquées ici.

Il est possible de détailler l'opposition entre sourciers et ciblistes selon trois instances. Les sourciers s'attachent au signifiant du texte original, ainsi qu'à la langue source dans laquelle il a été rédigé; et ils prétendent respecter scrupuleusement la langue source (Lo). Au contraire, les ciblistes mettent l'accent non pas tant sur le signifié que sur le sens ou - mieux sans doute, s'agissant de la traduction littéraire - sur l'effet du texte. Pour les ciblistes, au nombre desquels je me compte moi-même, il s'agit de traduire non pas la langue, mais la parole (au sens saussurien du terme), c'est-à-dire le texte, l'œuvre; et bien sûr traduire, c'est faire son deuil de la langue source, de toute évidence, pour mettre en œuvre toutes les ressources que nous offre la langue cible (Lt). Je ne traduis pas de l'allemand: je traduis des textes et des auteurs comme Nietzsche, Adorno ou Habermas, par exemple. Quand j'ai traduit Kant, j'ai pris congé de son «style», laborieux, lourd et emberlificoté, en m'efforçant de me mettre à l'école de l'aisance du français des auteurs du XVIII ${ }^{\mathrm{e}}$ siècle pour rendre son "Analytique du beau $»^{2}:$ c'était bien le moins! et c'était historiquement congruent.

En réalité, la position des sourciers revient à l'illusion littéraliste qu'on pourrait faire l'économie de la créativité en traduction, et même spécifiquement en traduction littéraire. Il n'y a qu'à traduire! entend-on parfois. Voilà une idée reçue à laquelle cèdent souvent ceux qui ne connaissent rien à la traduction. C'est le degré zéro de la créativité a contrario, que je viens de mentionner comme le déni de la créativité en traduction. 
Plus grave: le littéralisme est peut-être notre impensé de la traduction, dont la critique reste toujours à faire, pour autant qu'elle ne cesse de faire retour inopinément au détour de tel ou tel moment de fléchissement, de paresse ou d'inattention intellectuelle. Comme si la traduction n'était qu'un transcodage, une sorte de reproduction quasiment photographique du texte original (To). Alors que, comme nous le savons, la traduction est bien plutôt une re-production sur nouveaux frais (et j'écris « re-production» avec un trait d'union qui, en l'occurrence, serait plutôt un trait de désunion).

Il est encore bien d'autres sources à l'illusion sourcière. Il y a bien sûr le mirage de la traduction automatique (TA), de la «machine à traduire»; et a-t-on jamais vu qu'une machine, même perfectionnée, pût être créative?

Le littéralisme en traduction remonte aussi à un artefact pédagogique, tenant au fait que la traduction est utilisée (détournée?) comme dispositif d’apprentissage des langues étrangères et fait corrélativement l'objet de procédures d'évaluation qui tendent à la réduire à ce qu'Anna Svenbro a joliment appelé la «traduction-traquenard $»^{3}$.

Mais il y a plus. Depuis quelques décennies, une esthétique littéraliste de la traduction a reçu le renfort de penseurs de haute volée comme mes amis personnels et adversaires théoriques, les regrettés Henri Meschonnic et Antoine Berman. Je les range parmi les sourciers, même s'ils s'en défendent et récusent toute classification de cet ordre. Quoi qu'il en soit, les arguments qu'ils mettent en avant dans leur théorie de la traduction, et tout particulièrement dans les critiques qu'ils font de certaines traductions, ne laissent point de doute à mes yeux et confirment ma façon de voir les choses à ce propos.

L'un et l'autre sont de véritables penseurs et des auteurs de grand talent. Mais ils se trompent! à mes yeux. Dans le cadre de la controverse qui nous rassemble, je dirai que je ne fais pas montre de la même puissance argumentative qu'eux. Pour affirmer les positions qui sont les leurs, il leur faut beaucoup d'intelligence et de culture, de finesse littéraire et de puissance réflexive. Il leur faut bien ça pour défendre les positions fausses qui sont les leurs! alors que moi, je ne fais qu'avancer des paroles de bon sens. Je m’en tiens à énoncer la vérité toute nue qui, il est vrai, peut avoir ses charmes... Mais cela reste prosaïque au regard des discours ambitieux et séduisants des sourciers, dont on comprend bien le pouvoir de fascination qu'ils peuvent exercer chez certains. Il reste que la séduction intellectuelle et littéraire qui émane de leurs travaux mérite à mes yeux d'être entendue au sens étymologique, où "séduire» signifie plaire, mais aussi détourner, tromper, faire tomber dans l'erreur.

\section{II.}

À titre paradigmatique, je m'en tiendrai au concept de rythme, qui revêt l'importance que l'on sait chez un Meschonnic (1982). À le lire, le rythme est tout dans la langue poétique, et c'est cela qu'il incombe au traducteur de rendre. À mes yeux, cette idée holiste du rythme mérite d'être critiquée. D’abord, le rythme n'est qu'un aspect des choses: la musique de la langue qu'orchestre la parole poétique et littéraire va bien au-delà. J'accorde une importance au moins aussi grande à toutes les autres facettes du langage, comme la couleur des voyelles, la texture des consonnes, la tonalité des mélodèmes de phrases, la musique phonétique d'ensemble d'une langue, autrement dit sa «base articulatoire» (Malmberg 1964: 82 sq) ${ }^{4}$. Sans parler de ce que Barthes (1981) a appelé joliment le "grain de la voix». 
Pour subsumer tout cela, j'entends mettre en avant le concept de vocalité. Je parlerai volontiers aussi de la chair des langues et, de fait, il y a un érotisme propre à la vocalité de chacune d'elles (Ladmiral et Lipiansky 2015: 77-94, speciatim 89 sq., ainsi que 297 sq). C’est dans cette plénitude de la vocalité intériorisée dans la langue que s'opère ce que je me plais à appeler la suture de l'âme et du corps, en écho à une réminiscence lointaine à Saint-Augustin. On conviendra qu'il n'y revient au rythme qu'une part limitée, importante, mais limitée. Le rythme, c'est pour moi le squelette de la langue; et je préfère encore toute la chair dont il est la structure. Et le fait de mettre l'accent exclusif, massif, sur le rythme me fait penser mutatis mutandis à quelqu'un qui, au lieu d'avoir une photo de la femme aimée dans son portefeuille, n'aurait d'elle qu'un cliché radiographique: il serait assuré qu'elle a une image thoracique normale (ITN); mais moi, ce que je veux, c'est elle tout entière!

Que reste-t-il de tout cela (pour reprendre le refrain chanté par Charles Trenet) dans la traduction? La rétentivité esthétique des sons de la langue est, telle quelle, intransportable. Plus précisément: ce sont les sons qui, en eux-mêmes, sont objectivement intransportables d'une langue à l'autre; quant à la rétentivité esthétique, elle relève de la subjectivité et il reviendra au traducteur littéraire de talent d'en recréer en langue cible les effets pour le lecteur de l'œuvre. Outre ses limites intrinsèques, la phonostylistique ne saurait franchir les barrières de la traduction, comme l'a rappelé fortement Mounin (1986: 9-16). Ce qu’on traduit, ce ne sera jamais la phonétique mise en œuvre dans un poème, mais les effets symboliques et littéraires qu'elle induit. Il est illusoire de vouloir importer les signifiants d'une autre langue, d'en «singer le signifiant»- que ne puis-je écrire le singifiant! Ce serait céder à une illusion translinguistique relevant de l'erreur philosophique fondamentale qu'est à mes yeux le fantasme objectiviste.

Or, je dirai que le privilège exclusif accordé au rythme participe de cette même illusion philosophique. Comme si le rythme représentait quelque chose de «dur» dans le texte, capable de résister à la traduction. Comme s'il pouvait constituer une sorte d'invariant translinguistique. Pour tout dire: s'il s'agissait d'un morceau de musique, on ne garderait que la «section rythmique» en renonçant aux autres instruments et à la mélodie elle-même.

L'objectivisme conduirait ici à l'essentialisation sourcière d'une ontologie $d u$ rythme. Mais si la traduction du rythme est l'essentiel, et s'il revient à ce dernier le privilège ontologique de braver le décalage interlinguistique - en sorte qu'il serait possible de retrouver dans le texte cible ce même rythme - alors c'est qu'en l'espèce la créativité serait minimale et tendrait vers zéro. Encore une fois, cette mouture esthétique sourcière de l'«illusion de transparence» traductive (Ladmiral 2010a: 214, 241 et passim) correspond à ce que j'ai appelé la créativité a contrario (cf. supra). À dire vrai, et c'est un débat que j'ai eu avec Jean-Yves Masson, le «rythme» prend chez Meschonnic un sens élargi. Mais si ledit rythme englobe tout "ce qui va avec», alors ce n'est plus le rythme! C'est l'ensemble de ce qui fait la littérarité d'un texte. Comme on dit maintenant, c'est une question de "sémantique», qui permet la subreption des concepts. Sur la base de ce flou, tout le monde peut en tomber d'accord... 
III.

Plus généralement, l'approche sourcière en traduction débouche sur d'autres glissements, pas seulement sémantiques, mais d'une problématique à une autre. Ainsi la problématique esthétique du littéralisme va-t-elle dériver vers une problématique interculturelle, voire interlinguistique ${ }^{5}$.

Laurent Lombard ${ }^{6}$ nous a confié qu'il se réjouissait que certaines traductions accueillissent ce qu'il a appelé joliment «des copeaux de langue étrangère», où il voyait des «embrayeurs de l'imaginaire» qui bousculent la belle ordonnance du langage et sont de nature à faire advenir l'étrangeté foncière de la littérature. Ce sont là des paroles plaisantes et convaincantes qui, à première vue, semblent bien plaider pour le littéralisme d'une sensibilité sourcière. Mais il est possible d'en faire une autre lecture. En fait: si ces fameux «copeaux» de langue source restent des empreintes d'une langue vraiment étrangère au sein du texte cible d'une traduction ( $\mathrm{Tt}$ ), alors ce ne sont que ce que les linguistes appellent des xénismes, c'est-à-dire des fautes de langue. Ils n'acquièrent leur puissance évocatrice et leur valeur littéraire que d'être assimilés au sein de la langue cible (Lt), de s'y trouver comme naturalisés, et d'y fonctionner comme des éléments endogènes.

Il ne revient pas à la traduction littéraire de violer la langue cible, mais d'en faire lever des virtualités qui y étaient pour ainsi dire en attente, en latence, comme je l'ai montré en quelques occasions (Ladmiral 2015: 25 sqq. et passim). C'est ainsi, en y faisant entendre des accords nouveaux, comme d'un instrument de musique dans les mains d'un virtuose, qu'elle parvient à nous faire éprouver le bonheur esthétique de la lecture, en quoi réside proprement l'essence de la littérature. Or là, on n'est plus du tout dans l'illusion sourcière d'un littéralisme important la langue source dans la traduction. On a affaire, au contraire, à l'approche cibliste dans toute sa clarté. La traduction littéraire est bel et bien une création, mais sur le mode discret du talent. Ainsi la traduction cibliste s'attache-t-elle aux dessous de la créativité qui semblent d'abord cacher ce qu'elle finit par laisser voir (cf. supra).

Cela dit, on ne peut pas avoir le beurre et l'argent du beurre! sans parler du reste... Concrètement: en traduction, il arrive souvent qu'il n'y ait guère d'équivalent exact à l'item source enraciné dans sa langue-culture: X(LCo). On ne trouve pas l'équivalent cible dans la langue-culture de la traduction: $\mathrm{X}^{\prime}(\mathrm{LCt})$. Faute de traduction «directe», il faudra alors choisir entre deux (ou plus) traductions « obliques», plus ou moins approximatives: $\mathrm{Y}(\mathrm{LCt})$ ou Z(LCt). J'ai conceptualisé cette difficulté de traduction dans les termes du théorème de dichotomie! Un exemple trivial permettra de l'illustrer: pour traduire l'anglais source you, il faudra bien en français cible choisir entre tu et vous. Mais si j'ai cru bon de formuler un tel théorème, c'est que les exemples n'ont pas toujours cette simplicité. Il faut partir d'exemples simples, et même simplistes, pour conceptualiser de véritables problèmes de traduction, qui nous mettent en difficulté dans notre pratique de traducteur?

Du théorème de dichotomie, j'ai décliné plusieurs versions. Celle dont j'entends traiter maintenant concerne la traduction littéraire et le problème d'identité culturelle auquel elle se trouve parfois confrontée. Mais, auparavant, j'entends évoquer un autre de mes "théorèmes pour la traduction»: le théorème de finitude ou théorème de la finitude traductive, c'est-à-dire le théorème de notre finitude de traducteurs, qui est un principe qu'explicite la formule suivante: en traduisant, qu'est-ce que j'accepte de perdre? 


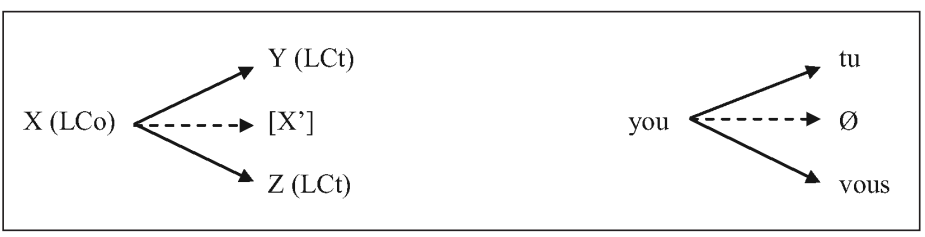

IV.

On s'entend dire souvent que telle traduction d'une œuvre littéraire ne respecte pas l'altérité culturelle de l'original. Certes, il convient de "garder» cette part d'étrangeté qui contribue à l'originalité d'une œuvre littéraire et y donne son prix. Mais il faut d'abord en revenir à la question cardinale: quand on traduit, qu'est-ce qu'on traduit? À vrai dire, c'est là une autre formulation, comme principe exotérique, de ce que j'ai thématisé d'une façon peut-être un peu ésotérique par le concept de quodité traductive (Ladmiral 2010a: 23 sq. et Ladmiral 2015: 150). Traduit-on l'altérité culturelle du texte original? ou traduit-on l'effet esthétique du texte? À quoi tient la singularité créative d'une œuvre, qu'on peut se plaire à appeler son étrangeté? Est-ce vraiment l'étrangeté de son identité culturelle d'origine? N'est-ce pas bien plutôt l'effet d'étrangeté qui tient à l'œuvre elle-même? à ce «frisson nouveau» dont elle est porteuse?

Poser la question, c'est y répondre. Je tiens que la traduction nous amène à bien marquer la différence entre l'esthétique littéraire propre à l'œuvre à traduire, d'une part, et les problèmes de communication interculturelle qu'elle pose occasionnellement, en raison de la distance plus ou moins grande avec la langue-culture dont elle provient et qui la sous-tend (LCo), d'autre part. Le traducteur se trouve face à un choix qui correspond à l'une des variantes que j'ai conceptualisées de mon «théorème de dichotomie». Il devra décider si, dans sa traduction, il fait du texte original plutôt une CEuvre cible ou plutôt un document cible.

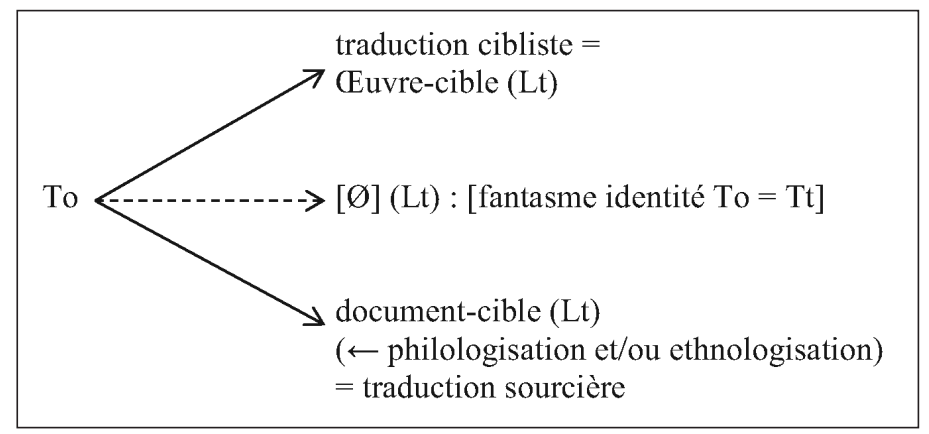

Plus fondamentalement, on a là l'illustration de l'une des problématiques sur lesquelles débouche l'immémoriale question du littéralisme, aux termes de laquelle s'opposent sourciers et ciblistes. Dans une perspective qu'on pourra dire classique, la démarche cibliste entend se centrer sur le projet de l'auteur qu'a mis en œuvre le texte à traduire. Pour nous, il s'agit essentiellement d'une œuvre littéraire. Mais ce peut être aussi un discours philosophique, un manifeste politique, une argumentation juridique, une déclaration oratoire, un texte scientifique, etc. Au-delà même d'une typologie de la traduction, à laquelle je ne fais ici qu'une allusion cursive, c'est cette 
spécificité propre au texte qu'il convient de traduire. En revanche, pour certains sourciers, la traduction devra surtout relever le défi du décrochement interculturel qu'implique l'accueil du texte source (To) dans la langue cible et la périlangue culturelle qui y est attachée (Ladmiral 2010a: 61, 178 et passim).

Dès lors, le texte cible (Tt) devient un document ethnoculturel, et n'est plus que secondairement une œuvre littéraire. Mais, parallèlement à cette ethnologisation de la traduction, il peut y avoir aussi une variante linguistique de cette communication interculturelle à laquelle est assimilée la traduction: on procède à une sorte de philologisation du texte qui prétend faire entendre quelque chose (?) des signifiants de la langue source dans le texte cible. Le texte à traduire tend à devenir un simple "prélèvement» linguistique (ou idiomatique), au mépris de ce qu'a pu être le projet de son auteur. Dans des cas de ce genre, et compte tenu de l'accumulation des connaissances disponibles, on sera porté à préférer l'érudition savante à l'appréciation esthétique du texte. Et là, pour le coup, on est bien dans une dénégation sourcière de la créativité littéraire de la traduction, a contrario donc!

\section{V.}

Mais le pire dans ces deux versions du littéralisme sourcier que constituent la version culturelle d'une ethnologisation de la traduction et la version linguistique d'une philologisation de ladite traduction, ce sont sans doute les dérives idéologiques auxquelles elles prêtent le flanc. Il y a là quelque chose de tout à fait paradoxal à voir ainsi le clivage opposant sourciers et ciblistes réinvesti par l'idéologie et devenant par là objet de controverses, de "disputes», dont la véhémence a vraiment de quoi surprendre. C'est précisément la véhémence des polémiques déclenchées à l'occasion de mes premiers textes sur le couple «sourcier et cibliste» qui m'a, pour ainsi dire, obligé à y revenir à plusieurs reprises et finalement à y consacrer tout un ouvrage (Ladmiral 2015: speciatim 3-27 et XI sq.).

Qu'on en juge! Dans leur critique des ciblistes (et corrélativement dans leur apologie tacite des sourciers), des auteurs respectables comme Meschonnic et Berman ne craignent pas de se laisser aller à des débordements polémiques dont la virulence n'est pas que de forme, mais concerne le contenu même de leur «théorie». Pour Meschonnic (1973), la position cibliste ressortit à une linguistique colonialiste! et ici, l'idéologie rejoint l'a priori proprement politique. Quant à Berman (2008: 48), il stigmatise la traduction cibliste comme traduction ethnocentrique! et là, l'idéologie prend une dimension anthropologique, plus profonde et en un sens encore plus critiquable à mes yeux. Cela dit, à bien y réfléchir, les engagements politiques de la plupart des intellectuels relèvent sans doute de ce même présupposé anthropologique tacite.

Mais, dans cette affaire, que devient réellement la traduction? et surtout, que reste-t-il de la créativité littéraire qu'implique une grande traduction? Il y a là une contamination idéologique de l'esthétique littéraire. Pire! cela ne concerne pas seulement la traduction (littéraire). Au-delà du tournant idéologique qu'on voit poindre en traduction ${ }^{8}$, on assiste plus généralement à l'extension d'un phénomène analogue au sein de la critique littéraire, qui ne va que trop souvent subordonner l'appréciation esthétique d'une œuvre à des critères idéologiques plus ou moins avoués. Au reste, ces critères sont assez larges et nombreux; mais je m'abstiendrai ici d'en donner une liste, pour ne pas me «mettre en danger» en contrevenant au politiquement-correct. 
Peut-être l'exercice d'un jugement esthétique est-il trop délicat, trop risqué pour d'aucuns qui, dès lors, préfèrent se replier sur les positions moins exposées d'un discours dominant et balisé...

Il en va de même dans l'art conceptuel contemporain (je serai tenté de l'appeler, de par une double apocope, l'art con-con!) qui s'autorise surtout d'un très abondant discours idéologique abscons que nous tient l'«artiste» sur ses productions et préalablement à ces dernières (à moins que ce discours ne tende à prendre la place desdites «productions»... $)^{9}$. Toujours est-il qu'en matière littéraire le lynchage dont a été victime un écrivain comme Richard Millet est très significatif du panurgisme idéologique et de l'acrimonie de certains auteurs comme Annie Ernaux, pourtant mieux inspirée dans certains de ses livres, que j'avais lus avec intérêt. Richard Millet est sans doute une personnalité incommode, mais c'est un grand écrivain, c'est un écrivain ${ }^{10}$ ! On constate un effet semblable de censure pour le cinéma, avec un supplément préalable d'autocensure en raison des conditions économiques tenant au fait que le septième art est aussi (et d'abord?) une industrie. Je prendrai trois exemples. La critique a boudé le très beau film de Rohmer L'Anglaise et le Duc (2001). Il y a même eu une censure idéologique caractérisée dont a fait l'objet le film magnifique comme un western social Cristeros (mis en scène par Dean Wright en 2012); et plus encore Katyǹ, qui est une œuvre plus difficile, par laquelle le grand Wajda aura sans doute terminé et couronné son admirable carrière cinématographique: ce dernier film a été carrément boycotté!

Quoi qu'il en soit, le problème est à mes yeux beaucoup plus fondamental. Parmi les diverses problématiques sur lesquelles débouchent paradoxalement les débats touchant le clivage sourciers/ciblistes, il y a effectivement une problématique idéologique dont je viens d'esquisser les linéaments. Mais je poserai en principe que la polémique idéologique renvoie plus profondément à l'anthropologie d'un impensé politique sous-jacent à la modernité occidentale. Pour le dire carrément: je vois là le travail négatif de déconstructions culturelles multiples relevant d'un ethnomasochisme généralisé qui grignote l'identité de nos sociétés. Pour reprendre une catégorie nietzschéenne et heideggérienne, j'y vois une des figures du nihilisme moderne et, plus concrètement, le symptôme d'une haine de soi qui fait l'objet de projections sur d'autres que soi. Je suis bien conscient que ce que j'avance ici est massif et mériterait d'être argumenté. Les limites imparties à la présente étude m'imposent de m'en tenir à présenter la chose comme un postulat ${ }^{11}$.

On comprend mieux dès lors la violence des polémiques. Ainsi la traduction n'est-elle pas à l'abri d'un retour à l'irrationnel sur le mode de la régression idéologique. Les sourciers «reprennent du service» : le cibliste devient un adversaire politique à combattre, les positions qu'il défend sont discréditées parce que condamnables! L’ethnocentrisme attribué au cibliste est disqualifié comme élitiste et conservateur, archaïque et réactionnaire. Là-contre, je conteste le terme même de traduction « ethnocentrique», dont la connotation est idéologiquement polémique, et je propose de lui substituer la formulation plus neutre et descriptive de traduction ethnocentrée, voire idiocentrée. Quand je traduis, je respecte la langue cible dans laquelle j'écris (le français) et le public des lecteurs pour lequel je travaille à ma traduction. Pas plus dans mes traductions que dans mes propres textes, je n'entends être «dérangeant», pour reprendre un terme à la mode et valorisé par certains. À mon sens, le traducteur doit «tenir son lecteur par la main» : ce n'est pas au lecteur de faire l'effort, mais au 
scripteur (traducteur ou auteur) de travailler son texte pour le rendre lisible. C'est ce que je me plais à thématiser cum grano salis dans les termes du théorème du camembert paradoxal, à savoir: un bon texte (et notamment le texte cible d'une traduction), c'est à la fois un texte qui «coule» et un texte qui «tient»! (Cela ne va pas contre l'axiome de la non-imbécilité du lecteur, bien au contraire.)

La traduction est une modalité centrale de la communication interculturelle; et dans l'esprit de l'impensé idéologique que je dénonce ici, il semblerait qu'il faille décentrer la traduction vers la culture de l'autre: c'est la version interculturelle du littéralisme sourcier. Là encore, je dois me contenter de poser des principes. Pour le dire rapidement, le littéralisme sourcier relève de la "haine de soi», qui va de pair avec une idéalisation de l'autre. Pour brocarder cette fascination pour «l'autre», je me plais à user d'un néologisme cocasse et à la qualifier d’^altérophilie! où le suffixe «-philie» consonne avec les entités psycho-pathologiques comme la pédophilie, la zoophilie, la coprophilie, etc. Au reste, cette folie est aussi une sottise: vouloir donner à l'autre de l'altérité, c'est lui donner ce qu'il a déjà...

Au lieu de cela, je plaide pour une relation équilibrée à l'autre. En un mot, sur le plan interculturel, j'adhère entièrement à une vérité de bon sens énoncée par le grand historien Braudel: ce que nous pouvons donner de mieux au monde, c'est nousmêmes. Et j'ajouterai que nous n'avons que ça à donner! Il faut seulement se ressouvenir que l'autre est aussi quelqu'un. Le grand africaniste Bernard Lugan nous rappelle que l'Afrique subsaharienne n'est pas peuplée d'Européens pauvres à peau noire! mais d'Africains, qui ont leur identité, leurs identités (au pluriel). On rejoint ici les réflexions philosophiques contemporaines, en vogue outre-Atlantique, sur l'échéance relationnelle de la reconnaissance. C'est à ce prix-là que le dialogue interculturel devient possible par la réciprocité, sans les messianismes conquérants que l'on connaît, et que le traducteur littéraire pourra chercher plus modestement à retrouver son propre chemin vers la créativité qui, pour être discrète, n'en est pas moins pour autant réelle (cf. supra) ${ }^{12}$.

\section{VI.}

Avant de conclure, je voudrais revenir sur la troisième facette de la créativité que j'ai évoquée au début, à savoir: l'horizon aporétique de la créativité. En cela, je ne fais que prolonger la belle conférence inaugurale qu'a donnée Lance Hewson à l'orée de notre colloque (le 20 mai 2015) ${ }^{13}$ sur les neuf paradoxes de la créativité en traduction littéraire, tout en restant peut-être un peu en deçà.

Traitant de la créativité, on se heurte d'emblée à une aporie - par construction, comme disent les géomètres. Que peut-on dire de la créativité? N'est-on pas condamné à n'en rien pouvoir dire? De fait, le paradoxe fondamental et insurmontable est qu'il s'agirait de parler de ce qui n'est pas encore. Ou bien: on ne peut pas parler de ce qui n'est pas. Ou bien : si on peut en parler, c'est qu'elle est déjà là; mais alors, ce n'est plus vraiment de la créativité. Ce paradoxe prend ainsi la forme exacerbée d'une antinomie, c'est-à-dire: deux propositions simultanément vraies et contradictoires, ce qui en toute rigueur est impossible. Cette aporie est la rançon d'une exigence élevée. Pour nous, en effet, la créativité fait figure d'idéal à l'horizon de l'écriture traduisante.

Mais on peut voir les choses autrement et redescendre à des considérations plus prosaïques. Il y a aussi un mauvais côté de ladite "créativité», qui peut prendre dif- 
férentes formes dégradées. Jadis, à l'école des Beaux-Arts, on apprenait le métier: on apprenait les «techniques» de la sculpture, de la peinture... Maintenant, on fait «de la créativité», c'est-à-dire n'importe quoi! et les étudiants artistes en reviennent, désorientés et découragés. On n'est jamais créatif que sur le fond d'une véritable formation, d'un métier qu'on a acquis. Autre chose: qu'est-ce qu' « un créatif»? Où y a-t-il des créatifs? dans les agences de pub! C'est tout dire. Sans parler des «séances de créativité » organisées dans certaines entreprises. On se demande ce qu’on y fait. Tout cela nous ramène à un niveau d'insignifiance, qui n'est évidemment pas le nôtre. Au plus haut niveau: Sa Sainteté Benoît XVI a critiqué la créativité en matière liturgique; et il a publié un motu proprio le 7 juillet 2007 rétablissant la forme extraordinaire du rite tridentin autorisant la messe en latin: Summorum Pontificum cura.

D’une façon générale, le danger est que la créativité soit la tentation d'une dérive vers le n'importe-quoi. Mais quel est le rapport avec la traduction? J'y viens. La créativité en traduction, c'est à mes yeux le projet cibliste qui nous amène à nous désassujettir du signifiant source et à prendre le risque d'une réécriture du texte cible qui constituera une véritable traduction. En quoi consiste ce que j'appelle dans ma traductologie le théorème de dissimilation ${ }^{14}$. Cette créativité traductive s'inscrit dans le cadre d'une esthétique classique, où la forme est serve. À cet égard, la traduction nous fournit un paradigme du classicisme, qui a pour idéal de ciseler une forme qui soit la mise en œuvre rigoureuse du fond: le texte cible de la traduction s'assigne aussi la mise en forme rigoureuse, c'est-à-dire «fidèle», de ce fond auquel pourvoit le texte source de l'original.

Quelles sont les autres possibilités alternatives à ce juste milieu cibliste? Il y en a deux. Soit: on dérive vers une démarche ultra-cibliste, un peu comme les exemples aberrants de "créativité» qui viennent d'être évoqués. Avec pour effet: au mieux, lesdites «belles infidèles»; et au pire, des traductions exécrables dont les exemples ne manquent pas. Soit: on verse dans le littéralisme des sourciers dont j'ai d'emblée marqué qu'ils contribuent à un déni de la créativité, pour ainsi dire a contrario. J'y distingue plusieurs modalités. Par excès, certains vont de facto jusqu'à une sacralisation du texte source qui condamne le traducteur à minimiser sa part de créativité dans le texte cible de sa traduction. Le texte original est investi comme un texte originaire, dont la Lettre dicterait sa loi. Par défaut, le littéralisme sourcier sera souvent une forme de régression, soit que la maîtrise du texte source se révèle être insuffisante, soit qu'il y ait un déficit de créativité dans la rédaction du texte cible. Alors, on se figure qu'on va «limiter la casse» en se raccrochant à la lettre des signifiants source, comme s'il pouvait s'opérer une osmose interlinguistique d'un texte (To) à l'autre (Tt). En fait, on perd sur les deux tableaux: le texte cible obtenu sera en règle générale un mixte de qualité médiocre; et en plus, ça se voit! ça se lit, ça s'entend ${ }^{15} \ldots$

Cela dit, toute traduction littérale n'est pas nécessairement une mauvaise traduction. Et il arrive même qu'elle soit bonne (sinon la meilleure), comme l'a indiqué Lance Hewson. Nous avons tous fait l'expérience de chercher avec difficulté la bonne solution pour un passage difficile d'un travail de traduction que nous avons sur le métier; et ne voilà-t-il pas que, contre toute attente, on en vient à s'apercevoir que la traduction littérale convenait parfaitement. Mais c'est l'exception qui confirme la règle. Il y a là un double paradoxe. Ce n'est pas du côté de la traduction littérale que nous avons cherché d'abord: nous avons cherché à «dissimiler», à juste titre; et ce n'est qu'au terme d'efforts laborieux que nous avons envisagé un retour à la littéralité, 
de guerre lasse et en dernier recours. Si on était parti directement de la littéralité, on aurait perdu du temps ou fait une mauvaise traduction du texte (ou les deux!). Dans des cas comme ceux-là, il ne faut pas voir un déficit de créativité, à la différence de ce qui est la règle pour les traductions sourcières. Paradoxalement, on a là un cas de créativité "en creux», qui ne se situe pas tant sur le plan de la production d'une écriture traduisante que sur celui de la réception d'une lecture créative de cet énoncé cible inattendu.

Cela illustre l'Esthétique de la traduction (que j'écris avec une majuscule), avec l'acception que je lui confère en écho à une remarque de Kant et en reprenant le sens étymologique du terme grec $\alpha \ddot{l} \sigma \theta \eta \sigma \iota \varsigma$ (= aïsthèsis) qui a la signification générale de perception, de sensation. Au-delà des différentes modalités de l'esthétique littéraire des traductions, l'Esthétique de la traduction telle que je l'entends désigne l'attention portée au ressenti langagier du texte. D’abord: du texte original (To), avec toutes ses richesses qui, de fait, n'existent que par la grâce de leur réactivation dans la sensibilité du lecteur. Ensuite: du texte cible de la traduction (Tt), tant il est vrai qu'il conviendra de prendre garde à la dialectique du décalage qui pourra venir s'insinuer entre le vouloir dire (sans trait d'union) psychologique initial du traducteur dans sa tête et le vouloir-dire (avec trait d'union) sémantique terminal de sa traduction sur le papier (ou sur écran). C’est ce que je me suis plu à appeler le théorème du gâteau au four.

Au bout du compte, on a là des cas (exceptionnels) où ladite créativité a contrario doit s'entendre en un sens positif. Mais, encore une fois, c'est bien l'exception qui confirme la règle. En prenant de la hauteur, je résumerai mon point de vue sur la question en invoquant un principe auquel je tiens: les sourciers n'ont jamais raison - que pour des raisons ciblistes ${ }^{16}$ !

\section{VII.}

Comme il a été indiqué, le paradoxe de la créativité est qu'il serait illusoire et contradictoire qu'on pût la prévoir. Comme nous y rappelait Georges Brassens, dans un tout autre contexte: «ça s'commande pas»... Tout au plus sans doute peut-on être dans une attitude d'attention et d'attente qui lui permette d'advenir.

Et pourtant, on retrouve le problème au niveau élémentaire de la traductologie telle que j'ai entrepris de la thématiser. L'important n'est pas, à mon sens, de décrire la traduction une fois faite ( $\mathrm{Tt}$ ) et de la comparer avec le texte original (To) dont elle s'autorise. Pour reprendre une formule que j'affectionne: mon problème n'est pas de savoir comment un autre a traduit hier, mais comment moi, je vais traduire aujourd'hui. C'est la différence que je fais entre une traductologie descriptive, qui s'attache à un travail rétrospectif de comparaison contrastive entre le texte source et sa traduction (ou ses traductions), et la traductologie productive pour laquelle je plaide, dont le discours vise à anticiper la traduction à faire ${ }^{17}$.

Telle est la fonction que j'assigne à mes «théorèmes pour la traduction», qui se veulent des théorèmes pour traduire. Mais il est bien vrai qu'il y a sinon une contradiction, du moins une discordance à pointer la nature aporétique de la créativité (cf. supra) et, parallèlement, prétendre apporter des éléments qui permettent d'anticiper une traduction. Sans doute faut-il y voir un exemple de ce que les psychologues appellent la dissonance cognitive, qui est à mes yeux un contrecoup de la nécessité devant laquelle nous nous trouvons, ici et ailleurs, de prendre en compte la complexité 
du réel et de l'existence humaine, avec ses contradictions irrésolues, ainsi que nos ignorances.

Les théorèmes de la traductologie productive sont les concepts et les principes qu'elle va puiser dans l'interdisciplinarité des sciences humaines et dans la philosophie ${ }^{18}$ comme dans notre héritage littéraire et culturel, et qui lui permettront d'ouvrir à la production d'une écriture traduisante un espace de possibilité où ils ne constituent que les balises d'une désinhibition de la créativité minimale à laquelle ne peut pas ne pas tendre le traducteur. C'est peu, mais c'est beaucoup.

Dans un esprit à peine différent, j'entends conclure en évoquant la créativité minimaliste que je vois à l'œuvre dans l'écriture traduisante. Contrairement aux sourciers, qui se plaisent à bousculer la langue cible en la pliant aux particularités de la langue de l'original, le cibliste s'attachera au travail endogène de la langue dans laquelle il rédige sa traduction (Lt). On pourra dire qu'il en appelle à une créativité a contrario en un sens positif, qui en vient à laisser voir les dessous de la créativité (cf. supra). Le cibliste est un prince charmant qui vient donner le baiser de la traduction à cette Belle au bois dormant qu'était sa langue cible encore partiellement assoupie; et en l'éveillant, il fait lever en elle des possibles qui sommeillaient dans le jardin intérieur de ses virtualités captives, en sorte qu'elle nous dévoile le secret de ses charmes cachés, jusque-là méconnus.

Avignon, mai 2015

\section{NOTES}

1. Colloque La traduction littéraire comme création, Avignon, 20 et 21 mai 2015. Certains des articles de ce volume ont leur origine dans une communication présentée lors de ce colloque.

2. Il s'agit de l'une des sections de la Critique de la faculté de juger (Kant 1790/1985: 913-1299). Reprise et réédition en livre de poche à partir de 1989 chez le même éditeur, dans la collection «Folio essais», no 134 .

3. Ce sont des phénomènes que je me suis attaché à thématiser de façon savante comme l'interférence docimo-pédagogique (Ladmiral 2010a: 23-83, speciatim 69 sqq). Voir, plus généralement et parmi bien d'autres publications, les Actes d'un colloque récent: Medhat-Lecocq, Negga et Szende (2016), speciatim 1-10 et 179-187.

4. Plusieurs fois réédité, ce petit livre constitue une excellente introduction à la phonétique.

5. Je ne développe pas plus avant ici l'essentielle et vaste problématique d'une esthétique de la traduction, à laquelle j’ai déjà consacré quelques études (Ladmiral 2004, 2012a), et qui fera l'objet d'un prochain ouvrage. À quoi il convient d'ajouter mon livre italien (Ladmiral 2009). Par le fait, je serai amené à y revenir cursivement à la fin de la présente étude.

6. Voir note 1.

7. Voir Ladmiral (1998). J'ai exposé le théorème de dichotomie dans un contexte interculturel, mais il s'agit d'un théorème général qui concerne toute forme de traduction: il s'applique sur le plan strictement linguistique d'abord, mais aussi dans le domaine littéraire (qui nous intéresse plus spécifiquement ici). Il trouve même à s’appliquer à propos de l'usage de la terminologie par exemple, ainsi que je l'ai indiqué dans Ladmiral (2012b).

8. C'est un point que je mentionne dans Ladmiral (2016).

9. Je ne m'engagerai pas plus avant dans ce débat. Parmi les travaux qu'il y aurait lieu de citer, je renvoie à ceux d'Aude de Kerros, sur «l'art caché», ou de Christine Surgens, qui est notamment une merveilleuse polémiste, mais aussi à Jean-Louis Harouel (2009/2015).

10. On trouvera les éléments du dossier dans le livre de Muriel de Rengervé (2013).

11. Je ne peux que renvoyer les lecteurs à l'étude philosophique où j’ai esquissé une argumentation plus détaillée, Ladmiral (1989). Voir aussi Alexandre Del Valle (2014).

12. Voilà une illustration de l'adage "Être et savoir disparaître», sur lequel je conclus mes théorèmes (Ladmiral 2010a: 247). 
13. Voir note 1, et l'article de Lance Hewson dans ce même volume.

14. Ladmiral (2010a: 57, 190, 218...). Dans mon dernier livre (Ladmiral 2015), je fais amplement travailler ce concept dont on trouvera de nombreuses occurrences: 95 sq., 197-199 et passim.

15. Entre ces deux pôles, on trouvera tout un dégradé de variantes. Je renonce à développer ici plus avant cette problématique en détail, puisqu'aussi bien elle est omniprésente dans l'ouvrage (Ladmiral 2015) que j'y ai consacré.

16. J’y tiens tellement que je me suis laissé aller à le répéter plusieurs fois dans Sourcier ou cibliste (Ladmiral 2015).

17. C'est un enjeu essentiel sur lequel je suis revenu à plusieurs reprises: cf. notamment Ladmiral (2010b).

18. Cf. Jane Wilhelm (2012). On y trouvera pour ainsi dire l'organon méthodologique de Traduire: théorèmes pour la traduction (Ladmiral 2010a).

\section{RÉFÉRENCES}

Barthes, Roland (1951): Le Grain de la voix. Paris: Éditions du Seuil.

Berman, Antoine (2008): L'âge de la traduction: la tâche du traducteur de Walter Benjamin, un commentaire. Texte établi par Isabelle BERMAN avec la collaboration de Valentina Sommella. Saint-Denis: Presses universitaires de Vincennes.

Del Valle, Alexandre (2014): Le Complexe occidental. Petit traité de déculpabilisation. Paris: Éditions du Toucan.

Harouel, Jean-Louis (2009/2015): La grande falsification. L'art contemporain. Paris: JeanCyrille Godefroy.

KANT, Emmanuel (1790/1985): Critique de la faculté de juger. (Traduit par Jean-René LADMIRAL, Marc B. De Launay et Jean-Marie Vaysse) In: Ferdinand Alquié, Euvres philosophiques. Bibliothèque de la Pléiade. Vol. 2. Paris: Gallimard, 913-1299.

LADMiral, Jean-René (1989): Critique et métacritique: de Koenigsberg à Francfort? In: André Jасов, dir. L’Univers philosophique. Encyclopédie philosophique universelle. Vol. 1. Paris: Presses universitaires de France, 700.

Ladmiral, Jean-René (1998) : Le prisme interculturel de la traduction. Palimpsestes. 11:15-30.

LAdmiral, Jean-René (2004): L'Esthétique de la traduction et ses prémisses musicales. In: Gottfried Marschall, dir. La traduction des livrets. Aspects théoriques, historiques et pragmatiques. Musiques/Écritures. Paris: Presses de l'université Paris-Sorbonne, 29-41.

Ladmiral, Jean-René (2009): Della traduzione. Dall'estetica all'epistemologia. (Traduit par Antonio Lavieri) Modène: Mucchi.

Ladmiral, Jean-René (2010a): Traduire: théorèmes pour la traduction. Tel. Paris: Gallimard.

LADMIRAL, Jean-René (2010b) : Sur le discours méta-traductif de la traductologie. Meta. 55(1):4-14.

LADMiral, Jean-René (2012a): Esthétique de la traduction. In: Bénédicte de Buron-Brun et Franck Miroux, dir. Poétique \& traduction. Métaphrastiques. Reims: Presses universitaires de Sainte-Gemme, 9-25.

LADMIRAL, Jean-René (2012b) : La terminologie au risque de la traduction. In: Jean-Jacques BRIU, dir. Terminologie (II) : comparaisons, transferts, (in)traductions. Travaux interdisciplinaires et plurilingues. Berne: Peter Lang, 11-20.

LAdmiRal, Jean-René (2015): Sourcier ou cibliste. Les profondeurs de la traduction. Traductologiques. Paris: Les Belles Lettres.

LAdmiral, Jean-René et Lipiansky, Edmond Marc (2015): La Communication interculturelle. Traductologiques. Paris: Les Belles Lettres.

LADMIRAL, Jean-René (2016) : Un tournant épistémologique au fondement de la traductologie? In: Sylvie Monjean-Decaudin, dir. La traductologie et bien au-delà. Mélanges offerts à Claude Bocquet. Arras: Artois Presses Université, 303-315.

Malmberg, Bertil (1964): La Phonétique. Que Sais-Je? Paris : PUF.

Medhat-Lecoce Héba, Negga, Delombera et Szende, Thomas, dir. (2016): Traduction et apprentissage des langues. Entre médiation et remédiation. Paris: Éditions des archives contemporaines. 
Meschonnic, Henri (1973): Pour la poétique II: Poétique de la traduction. Le Chemin. Paris: Gallimard.

Meschonnic, Henri (1982): Critique du rythme. Anthropologie historique du langage. Lagrasse: Verdier.

Mounin, Georges (1986): Phonostylistique et traduction. Revue d'esthétique. 12:9-16.

Rengervé, Muriel de (2013): L'Affaire Richard Millet. Critique de la bien-pensance. Paris: Éditions Jacob-Duvernet.

Wilhelm, Jane (2012) : Jean-René Ladmiral - une anthropologie interdisciplinaire de la traduction. Meta. 57(3):546-563. 